\title{
Study of the dependence of organic additives on the properties of 3D-structured catalysts properties for hydrocarbon hydrotreatment
} \author{
Lysikov $^{2,3}$ \\ ${ }^{1}$ Novosibirsk State Technical University, 630073, Russian Federation \\ ${ }^{2}$ Boreskov Institute of Catalysis SB RAS, 630090, Russian Federation \\ ${ }^{3}$ Novosibirsk State University SB RAS, 630090, Russian Federation
}

N.S. Lazarenko ${ }^{1}$, N.D. Kaplin ${ }^{1}$, E.E. Vorobyeva ${ }^{2,3}$, E.V. Parkhomchuk ${ }^{2,3}$, and A.I.

\begin{abstract}
The dependence of porosity and durability of $\mathrm{Al}_{2} \mathrm{O}_{3}$ on various organic additives (ethylene glycol, diethylene glycol, glycerin, industrial oil and their combinations) was shown. Some of results were used to synthesize 3D-structured catalysts.
\end{abstract}

There is a collection of waste combustibles, as result of consumption of fossil fuels (gasoline and diesel). These wastes are low-quality energy sources due to their high viscosity, explosion point and large amounts of impurities based on heteroatoms: nickel, vanadium, sulfur, nitrogen, etc. It is impossible to use them directly as a fuel. It might be only used as boiler fuel to make the giant environment damage. Therefore, recycling these wastes is important task to produce fossil fuels.

There are 49 large-scale heavy crude refining units operating in the world with fixed catalyst bed and 22 with fluidized catalyst bed at the present time [1]. Most of them use the process of coking of crude oil, which produces both light fractions and massive coke particles due to disproportionation of hydrocarbons, partially burned out to supply heat to the process. However, this approach could be hardly called efficient. The output yield of fossil products only achieve about $30 \mathrm{wt} \%$. On the other hand, hydroprocessing processes of residue feedstocks could be more efficiant and they allow to clean the feedstock from impurities and increase fossil fuel yields up to $70 \mathrm{wt} . \%$. The process is made to a multistage sequential reactors to increase refining efficiency. Each stage is aimed at increasing the efficiency of specific refining stages: the first stage is carried out to purify from asphaltenes and metals, the next is carried out to remove of sulfur and nitrogen, and the last is completed by hydrocracking and isomerization processes [2]. This sequence is required to durable catalysts of separate stages at presence of high content of impurities in feedstock. At the first stage wide-porous $\mathrm{Al}_{2} \mathrm{O}_{3}$ catalyst is used in the process of removal asphaltenes and metal-organic particles ( $\mathrm{Ni}$ and $\mathrm{V}$ ). $\mathrm{Al}_{2} \mathrm{O}_{3}$ impregnated by metal oxides (W, Mo, Co, $\mathrm{Ni}$ ), is used at the second stage. And on the last stage, the catalyst are enhanced to improve the acidic properties by doping zeolites, such as Y or ZSM-5 [3].

$\mathrm{Al}_{2} \mathrm{O}_{3}$ synthesis is important task to produce a high efficient carrier for heavy oil refining. The preparation of $\mathrm{Al}_{2} \mathrm{O}_{3}$ should carried out high porous material. Conventional 
mesoporous $\mathrm{Al}_{2} \mathrm{O}_{3}$ has a smaller pore volume $\left(0.45 \mathrm{~mL} \cdot \mathrm{g}^{-1}\right)$, and it has the high initial activity, dramatically decreased at time. In addition to conventional $\mathrm{Al}_{2} \mathrm{O}_{3}$, the catalyst with a large meso- and macropores $\left(1.12 \mathrm{~mL} \cdot \mathrm{g}^{-1}\right)$ and hierarchical structure shows the huge stability. Fig. 1 clearly demonstrates pore blocking processes leading to complete catalyst deactivation of the conventional catalyst. At the same time the macroporous catalyst stays stable.
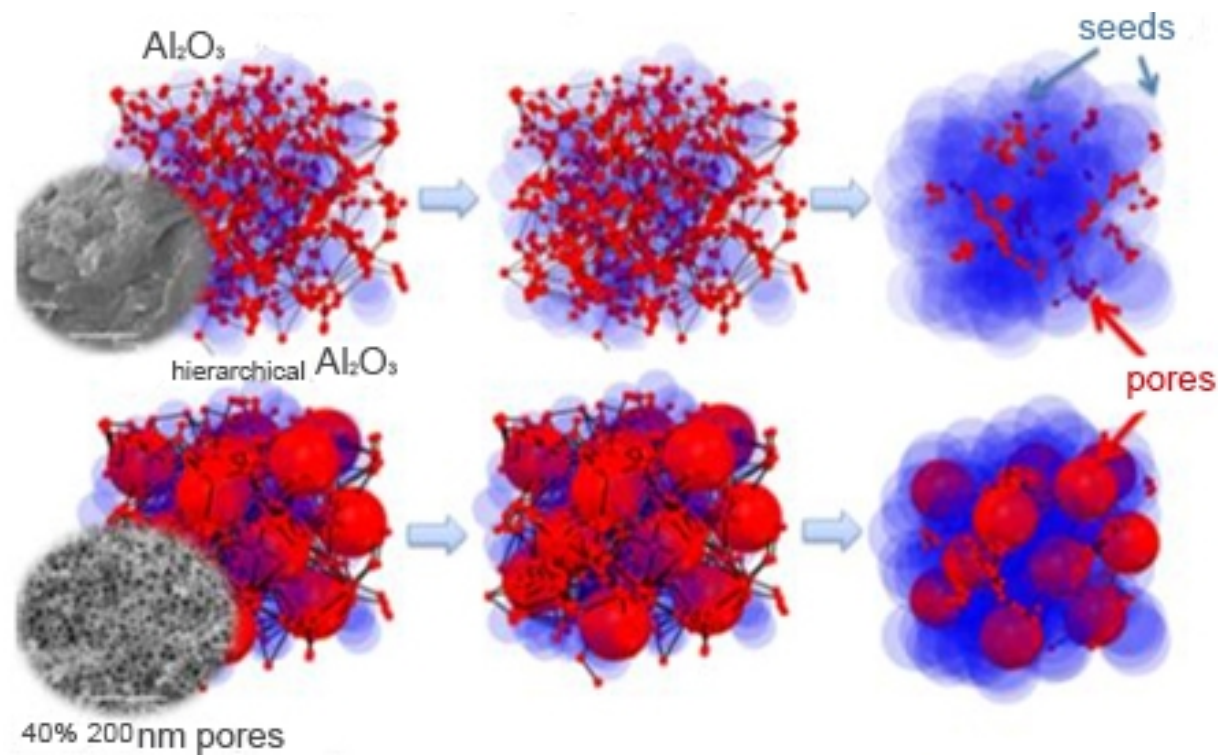

Fig. 1. Process of catalyst pore blocking in the asphaltene removing process due to coke formation.

Most of the methods of mesoporous catalysts preparation are based on extrusion of pellets and thermal control. But there are no any controls to macroporous size formation [4]. In addition to synthesis, the template additive easily makes the macroporous material [5]. The pore size could be controlled by this method in the huge range of $0.05-5.0 \mu \mathrm{m}$. Nevertheless, the external diffusion problem of viscous reagents could have a place for template alumina usage due to spaces between different pellets. The addition of inert particles such as $\mathrm{SiC}$ aims to avoid the problem in industry.

A better solution is to use another method to make the $\mathrm{Al}_{2} \mathrm{O}_{3}$ support, for example, by $3 \mathrm{D}$ printing technology. It is possible to fully control the geometry of the catalyst like as the reactor size by applying $3 \mathrm{D}$ printing and to achieve a minimal dependence of the external diffusion to process. Moreover, it could effect to reduce the hydrodynamic resistance of the bed, making all sections of the catalyst equally accessible to the feedstock.

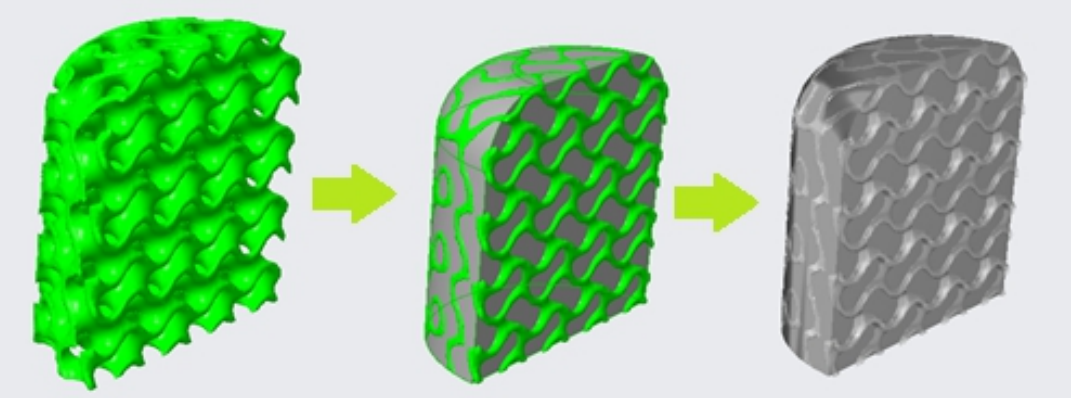

Fig. 2. Principal steps for fixing the designed catalyst with structured geometry. 
To prepare a catalyst with structured geometry was used the CAD systems (Solidwork, Creo, Autodesk). The model of catalyst was converted to STL file and subsequently printed by a Wanhao Duplicator D6 3D printer from PLA/ABS polymer to make a template. Then, the boehmite mixture, as an aluminum oxide precursor containing $20 \mathrm{wt} . \%$ polystyrene microspheres and various organic additives (diethylene glycol (Deg), ethylene glycol (Eg), glycerin (Gly), industrial oil (Oil) and their mixture), was introduced to the polymer template. Then, the precursor was dried and heated up to $700^{\circ} \mathrm{C}$ to remove the organic additives. The graphical scheme of catalyst conversion is shown in Figure 2. For comparison, the paste of the same carrier was formed to pellets with $3 \mathrm{~mm}$ diameter and 5 mm length.

The obtained support was analyzed by TPD of ammonia analysis. All samples prepared with the organic additives have lower acidity. It is interesting that the impregnations of organic additives increase the pore volume of alumina support. Moreover, the increase in pore volume is proportional to the viscosity and molecular weight of additives. Thus, for aluminum oxide with the addition of ethylene glycol and industrial oil, an increase in pore volume is up to $1 \mathrm{~mL} / \mathrm{g}$ as observed. The dependence of the pore volume on the additives is shown in Fig. 3.

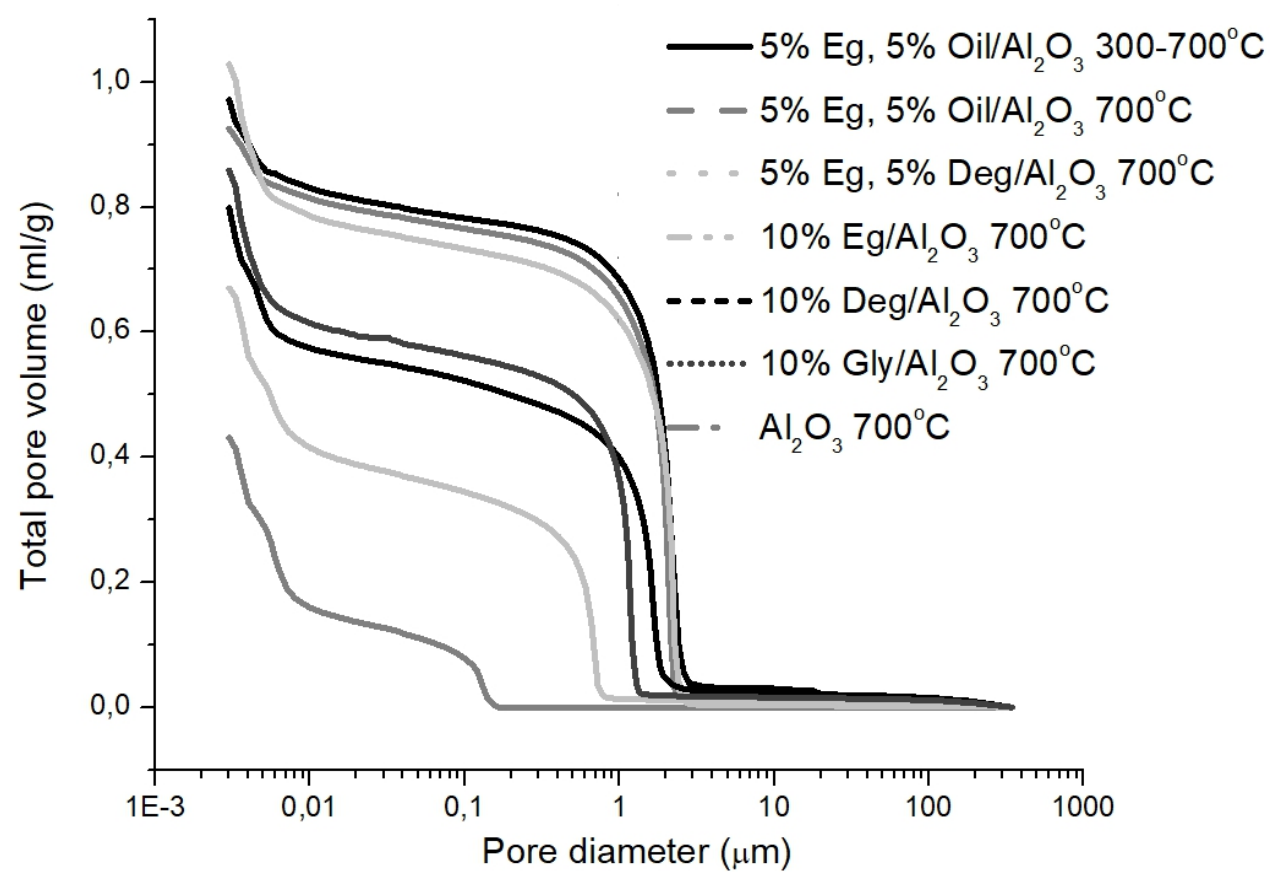

Fig. 3. Dependence of the pore volume on the additive used.

At the same time, average pore size for organic samples is more than $1 \mu \mathrm{m}$. It makes the obtained materials to be useful for hydrodemetallization and deasphalting processes of residue. SEM images of pore distribution are shown in Fig. 4. 


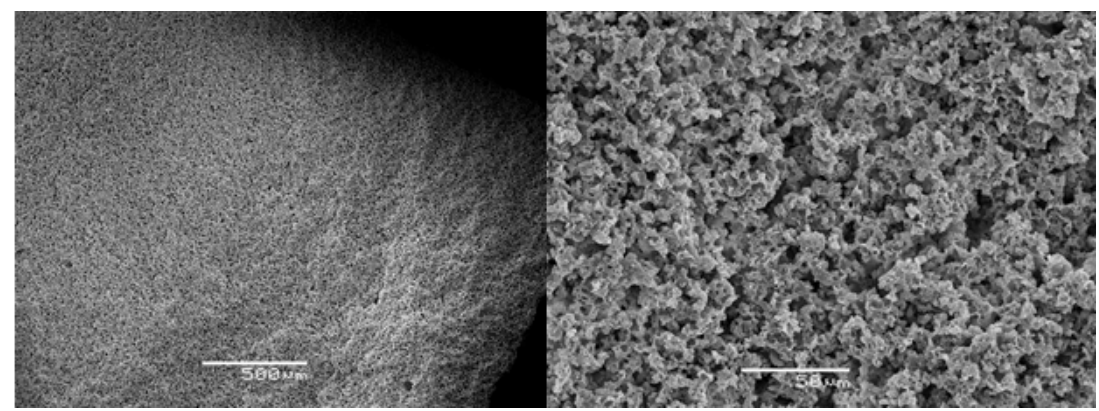

Fig. 4. SEM images of $\mathrm{Al}_{2} \mathrm{O}_{3}+10 \%$ EG.

Together with the change in the porosity of the sample, additives had a significant impact on the durability of the resulting pellets. These data should also be taken into account for the creation of an industrial catalyst. The obtained data on the effect of additives on the crushing strength of samples are shown in Fig. 5.

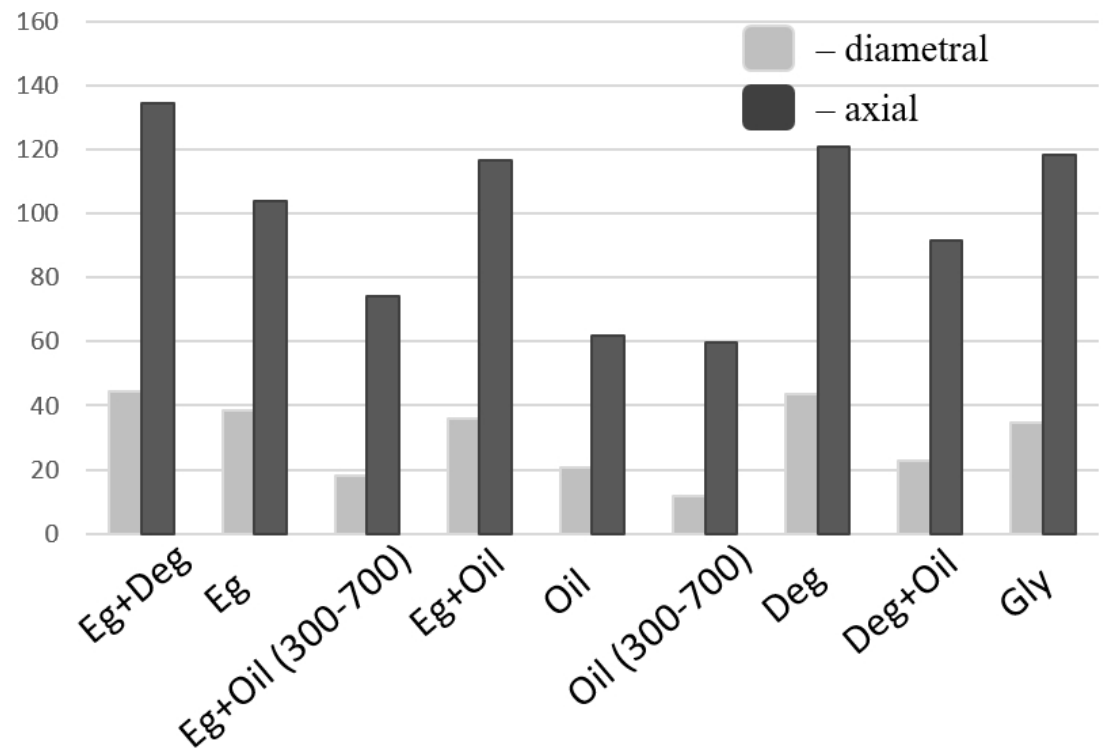

Fig. 5. Dependence of the mechanical durability on the additives.

Thus, new alumina supports have been made in this work. These materials is a suitable for catalytic processes of deasphalting and demetallization of heavy oils as shown. The possibility of support modeling makes possible to achieve the controlled catalyst layer. The organic additives have made different pore distribution and durability of material obtained.

This work was supported by the Ministry of Science and Higher Education of the Russian Federation within the governmental order for Boreskov Institute of Catalysis (project AAAA-A21121011490008-3). 


\section{References}

1. A. Mecke, I. Lee, J.R. Baker jr., M.M. Banaszak Holl, B.G. Orr, Eur. Phys. J. E 14, 7 (2004)

2. M. Ben Rabha, M.F. Boujmil, M. Saadoun, B. Bessaïs, Eur. Phys. J. Appl. Phys. (to be published)

3. F. De Lillo, F. Cecconi, G. Lacorata, A. Vulpiani, EPL, 84 (2008)

4. L. T. De Luca, Propulsion physics (EDP Sciences, Les Ulis, 2009)

5. G. Plancque, D. You, E. Blanchard, V. Mertens, C. Lamouroux, Role of chemistry in the phenomena occurring in nuclear power plants circuits, in Proceedings of the International Congress on Advances in Nuclear power Plants, ICAPP, 2-5 May 2011, Nice, France (2011)

6. E.V. Parkhomchuk, A.G. Okunev, A.I. Lysikov, P.D. Parunin, Russ. J. Uspekhi Chem. 9, 981 (2015)

7. E. Furimsky, Appl. Catal. A: General 2, 177 (1998)

8. A.V. Pashigreva et al., Catal. Today. 3-4, 164 (2010)

9. M. Antonietti et al., Adv. Mater. 2, 154 (1998)

10. E.V. Parkhomchuk,K.A. Sashkina, N.A. Rudina, N.A. Kulikovskaya, V.N. Parmon, Catal. Ind. 4, 23 (2012) 